\title{
References
}

\section{The Red Book of Varieties and Schemes}

1. Auslander, L., Mackenzie, R.E.: Introduction to Differentiable Manifolds. McGraw-Hill: New York 1963

2. Atiyah, M.: K-Theory. W.A. Benjamin: New York 1967

3. Bourbaki, N.: Commutative Algebra 1-7. Springer: Heidelberg 1988. Orig. publ. by Addison-Wesley, Reading, MA, 1972

4. Bourbaki, N.: Algèbre Ch. 8: Modules et Anneaux Semi-Simples. Hermann: Paris 1958

5. Bourbaki, N.: Algèbre Commutative 1-4. Reprint Masson: Paris 1985

6. Grothendieck, A., Dieudonné, J.A.: Eléments de Géométrie Algébrique I. Springer: Berlin-Heidelberg 1971

7. Grothendieck, A., Dieudonné, J.A.: Eléments de Géométrie Algébrique III/1. Publ. Math. IHES 11: Paris 1961

8. Gunning, R.C., Rossi, H.: Analytic Functions of Several Complex Variables. Prentice Hall 1965

9. Klein, F.: Vorlesungen über die Entwicklung der Mathematik im 19. Jahrhundert. Springer: Berlin 1926

10. Lang, S.: Introduction to Algebraic Geometry. Wiley-Interscience: New York 1958

11. Semple, J.G., Roth, L.: Introduction to Algebraic Geometry. Clarendon Press: Oxford 1985

12. Serre, J.-P.: Algèbre Locale. Multiplicités. Lect. Notes in Math. vol. 11. Springer: Berlin-Heidelberg 1975

13. Zariski, O., Samuel, P.: Commutative Algebra 1. Springer New York 1975

14. Zariski, O., Samuel, P.: Commutative Algebra 2. Springer New York 1976 